\title{
SHAPE PEELING FOR IMPROVED IMAGE SKELETON STABILITY
}

\author{
M.Spitzner ${ }^{*}$, R.Gonzalez ${ }^{*}$ \\ *University of Applied Sciences HTWD, ${ }^{\dagger}$ School of ICT, Griffith University
}

\begin{abstract}
This paper presents a novel hybrid preprocessing method for improving noise resilience and improved computational efficiency of image skeletonisation. Common techniques for extracting the topological skeleton of a shape include distance transforms, thinning, and geometric analysis. All of these methods are sensitive to noise, and can suffer from instability and unwanted spurs. In the case of needing to match skeletons from different images, instability can be a significant problem. Skeleton stability using the proposed preprocessing method is evaluated for a range of existing medial axis transforms. It is shown to be more effective for suppressing unwanted spurs and improving stability against other preprocessing techniques.
\end{abstract}

Index Terms - Skeleton, Medial Axis Transform, Shape Peeling, Stability

\section{INTRODUCTION}

Image skeletonisation is a key component in many applications of image processing and computer vision including image recognition, content-based retrieval structure analysis, object representation and visualization. Skeletonisation involves determining the medial axis of arbitrary shapes. A variety of medial axis transforms (MAT) have been developed for extracting skeletons from binary images that can be classified into four basic types.

Distance transform approaches were first introduced by Blum [1] and rely on calculating the nearest distance from each interior point to the boundary. In this approach, skeletons are defined as the ridges on the distance field. This can be calculated as the Euclidean distance or some other approximation [2] such as the Chamfer distance or by using fast marching methods [3]. While these are relatively quick and easy to calculate they tend to create unwanted spurs. Blum proposed reducing the spurs by considering only skeleton points whose radius function is greater than some given radius. Typically non-trivial post processing is required to eliminate the resulting spurs.

Morphological thinning approaches generate skeletons by iteratively removing pixels from the perimeter of a shape until the thickness of the resulting region is a single pixel. Many different approaches have been proposed in the literature [4]. Thinning algorithms are easy to implement and have the benefit of significantly reduced spur generation. They are also relatively fast, but slower than the distance transform approaches. However, they are not invariant to isometric transformations and they suffer from instability, as even minor changes to a shape boundary give rise to significant changes in the resulting skeletons.

Geometric approaches typically make use of Voronoi diagrams calculated from polygonal representations of the shape or a filtered set of points lying on the shape boundary [5]. The skeleton is obtained by pruning the resulting Voronoi diagram using heuristics. Due to preprocessing of the shape boundary, this approach is much less prone to instability from minor boundary variations. However the cost is significantly increased processing overheads due to both the preprocessing and the Voronoi diagram calculation which is itself an $O\left(n^{2}\right)$ worst case function.

These three basic approaches are sensitive to noise, resulting in the generation of unnecessary branches in the skeleton. Only general field function based approaches provide a degree of robustness. Typically some form of potential or electrostatic field function is used but flux fields can also be generated from the divergence of the gradient vector field of the Euclidean distance [6]. While this approach is more robust than the others it has significantly higher computational complexity [7].

In real-world image-processing tasks, image data may be captured under varying illumination, noise, and similarity transformations. This results in variations in the detected shape boundaries and translates into different skeletons being generated. This instability makes follow-on processing such as skeleton matching difficult. Accordingly a number of preprocessing techniques such as scale space filtering [8] have been proposed to reduce noise sensitivity.

This paper evaluates the stability of a variety of medial axis transform algorithms. It proposes, "shape peeling" for obtaining the medial axis transform of real-world image data that is stable and efficient under various scenarios. It assesses its effectiveness at improving stability and spur suppression against other preprocessing techniques.

\section{METHOD DESCRIPTION}

Common preprocessing techniques for improving skeleton stability include morphological processing and median filtering. These operate on local pixel neighbourhoods. While they reduce boundary noise somewhat they cannot take into account the wider characteristics of the boundary's shape. While it is possible to apply higher level geometric analysis to regularise shape boundaries as is done in the case of Voronoi based methods, this comes at a relatively high computational cost. A simpler alternative for regularising a shape's boundary that does consider the wider relationship between pixels and the 
rest of the shape is to peel off some of the shape's boundary by using the following algorithm:

Let $f(x, y)$ be an image where $[x, y] \in \mathbf{N}^{2}$. For a pixel $p$ with coordinates $(x, y)$ its 4 -neighbors is the set given by $(1)$ :

$$
\mathrm{N}_{4}(p)=\{(\mathrm{x}, \mathrm{y}+1),(\mathrm{x}, \mathrm{y}-1),(\mathrm{x}+1, \mathrm{y}),(\mathrm{x}-1, \mathrm{y})\}
$$

And its 8-neighbours is the set of pixels given by (2):

$$
\begin{array}{r}
\mathrm{N}_{8}(p)=\mathrm{N}_{4} \cup\{(\mathrm{x}+1, \mathrm{y}+1),(\mathrm{x}-1, \mathrm{y}+1), \\
(\mathrm{x}-1, \mathrm{y}+1),(\mathrm{x}-1, \mathrm{y}-1)\}
\end{array}
$$

Now with $\mathrm{K}$ being a large positive integer and $\mathrm{t}_{\mathrm{b}}$ being the minimum error threshold [9], let:

$$
h(p)=\left\{\begin{array}{cc}
0 & f(p)<t_{b} \\
K & \text { otherwise }
\end{array}\right.
$$

The minimum 4 and 8 -connected neighbours of $p$ are then:

$$
\begin{aligned}
& \mathrm{M}_{4}(p):=\min h(p): p \in \mathrm{N}_{4}(p) \\
& \mathrm{M}_{8}(p):=\min h(p): p \in \mathrm{N}_{8}(p)
\end{aligned}
$$

Now let $\bar{x}=\left\langle x_{i} \mid i<W\right\rangle$ and $\bar{y}=\left\langle y_{i} \mid i<H\right\rangle$ be finite sequences of natural numbers where $\mathrm{W}$ and $\mathrm{H}$ are respectively the width and height of $f$. Also let $\bar{p}:=\langle\bar{x}, \bar{y}\rangle$ be the ordered sequence of all pixels.

The algorithm is then:

$\forall \bar{p}, h(p):=\left\{\begin{array}{cc}M_{4}(p)+2 & ; M_{4}(p)<M_{8}(p) \\ M_{8}(p)+3 & \text { otherwise }\end{array}\right.$

Finally, letting $\bar{q}:=\langle|W-\bar{x}|,|H-\bar{y}|\rangle$ be the reverse ordered sequence, the following operation is performed:

$$
\forall \bar{q}, h(p):=\left\{\begin{array}{cc}
0 & ; M_{4}(p)+2<t_{d} \vee M_{8}(p)+3<t_{d} \\
K & \text { otherwise }
\end{array}\right.
$$

The value $t_{d}$ is a measure of the proximity of a pixel to the shape boundary and defines the thickness of the shape boundary to be peeled off. The selection of appropriate values for $t_{d}$, is dependent on the shape's dimensions.

The methodology used to assess the effectiveness of this preprocessing technique involved simulating the common processes that give rise to variations in shape boundaries of captured images. Variations in object and/or camera orientation were modeled in the form of isometric transformation. Additive white noise was used to model signal capture noise. Illumination changes were modeled as variations to the binary threshold used for foreground / background separation. These formed three separate test scenarios that were used to induce subtle changes in the shape boundaries from which the skeletons were obtained.

The effectiveness of shape peeling for improving the stability of seven different MATs was evaluated including:
- $\quad$ EDF - Euclidean Distance Field based skeleton

- $\quad$ ROS - Rosenfeld's parallel thinning method [10]

- $\quad$ PAV - Pavlidis thinning method [11]

- $\quad$ ZHA - Zhang \& Suen algorithm [12]

- $\quad$ GUO - Guo-Hall algorithm [13]

- $\quad$ FMM - Fast Marching Method [3]

- $\quad$ Flux - Flux field method [6]

Zhuo et.al. present a method for assessing the noisiness of shape skeletons [14]. This relies on the assumption that noisy skeletons result in a higher value in the sum of " $0 \rightarrow 1$ " transitions in the neigbourhood of each skeletal pixel. This metric however is not effective for evaluating the stability of the skeleton itself under subtle shape boundary changes. Instead for each skeleton produced, the number of branches and joins is counted. The coefficient of variation $c_{v}$ defined as the standard deviation divided by the mean is calculated for the number of branches and joins. Let $B_{i}$ denote the $\mathrm{i}^{\text {th }}$ branch and $J_{i}$ denote the $\mathrm{i}^{\text {th }}$ join in a skeleton. One can then define the coefficient of variation $c_{v A}$ for each test scenario with $A_{i} \in\left\{B_{i}, J_{i}\right\}$

$$
\begin{array}{cc}
c_{v A}=\frac{\sigma_{A}}{\mu_{A}} \\
\text { where } \mu_{A}=\frac{1}{N} \sum_{i=0}^{N} A_{i} \\
\text { and } \sigma_{A}=\sqrt{\frac{1}{N} \sum_{i=0}^{N}\left(A_{i}-\mu_{A}\right)^{2}}
\end{array}
$$

The effectiveness of a number of other preprocessing techniques for improving skeleton stability was similarly assessed. These included median filtering, morphological erosion, opening and closing. Erosion was used to smooth the shape boundary by removing pixels that protruded away from the boundary having less than four, 8-connected neighbours. Opening was used to erode all pixels having less than four neighbours and then dilate all having greater than four neighbours. The closing operator was used to dilate all having greater than six neighbours and then erode all having less than two neighbours.

\section{EXPERIMENTAL RESULTS}

A number of natural and synthetic images having simple shapes were obtained such as those in Figure 1. These images were rotated, had white noise added and where applicable, different thresholds were used to extract the foreground objects. In total, 36 different rotation angles were used, 36 different amounts of noise added in the range of 0 to $100 \%$ and where applicable 36 different global binary thresholds applied. Skeletons were obtained from the resulting images using each MAT without preprocessing. Figure 2 shows typical skeletons produced for some selected MATs in each row. The original image is on the left, with added noise in the centre and rotation on the right. 

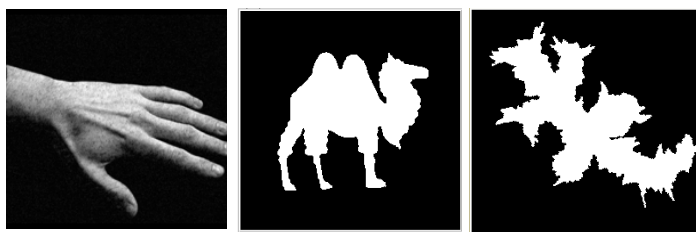

Figure 1 Typical Test Images

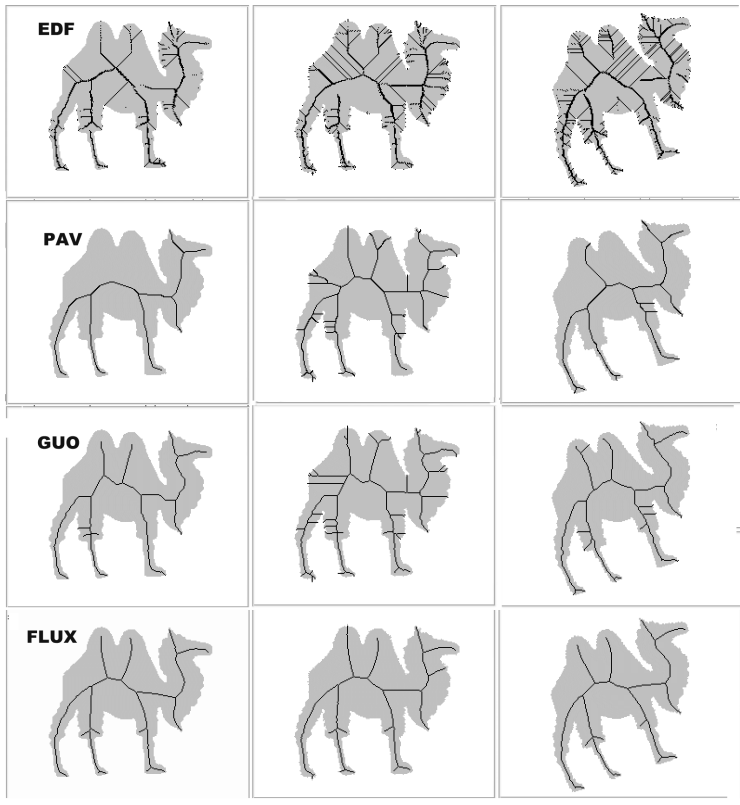

Figure 2. Basic Skeleton Stability

For each transform the number of joins and branches from the resulting skeletons across all images were counted and averaged and the coefficients of variation calculated as per equation (8) for the number of joins $\mathrm{C}_{\mathrm{vJ}}$ and branches $\mathrm{C}_{\mathrm{vB}}$. Table 1 shows that across all the test cases average, the coefficient of variation for most methods was above $30 \%$. All of the MATs were very sensitivity to additive noise. In general, the distance based methods such as the EDF and FMM were comparatively more stable than the others. However, while all the MATs produce spurious branches, the EDF is the worst by far. The FMM technique had the unfortunate tendency of producing disjoint skeletons unless manually adjusted.

\section{Table 1 Basic Skeleton Stability}

\begin{tabular}{|c|c|c|c|c|c|c|c|}
\hline & \multicolumn{2}{|c|}{ Rotation } & \multicolumn{2}{|c|}{ Threshold } & \multicolumn{2}{|c|}{ Noise } & Ave \\
\hline & $\mathrm{C}_{\mathrm{vB}}$ & $c_{v J}$ & $\mathrm{C}_{\mathrm{vB}}$ & $c_{v j}$ & $\mathrm{C}_{\mathrm{vB}}$ & $c_{v j}$ & $c_{v}$ \\
\hline EDF & 18.0 & 27.8 & 13.6 & 15.5 & 34.0 & 25.6 & 22.4 \\
\hline ROS & 14.9 & 17.3 & 24.4 & 27.3 & 61.8 & 65.1 & 35.1 \\
\hline PAV & 17.3 & 16.3 & 30.7 & 23.6 & 49.0 & 44.7 & 30.3 \\
\hline ZHA & 16.8 & 16.4 & 24.1 & 26.1 & 56.4 & 53.5 & 32.2 \\
\hline GUO & 15.8 & 15.7 & 25.8 & 25.0 & 51.3 & 50.4 & 30.7 \\
\hline FLUX & 25.2 & 24.7 & 10.0 & 10.2 & 65.6 & 48.9 & 30.8 \\
\hline FMM & 26.1 & 16.9 & 13.0 & 8.5 & 29.0 & 21.7 & 19.2 \\
\hline
\end{tabular}

Figure 3 shows the skeletons produced for one of the sample images for selected instances of each test case using morphological preprocessing (as given on each row) and the
Pavlidis method. From left to right is the original shape, with additive white noise, varying threshold and rotation.

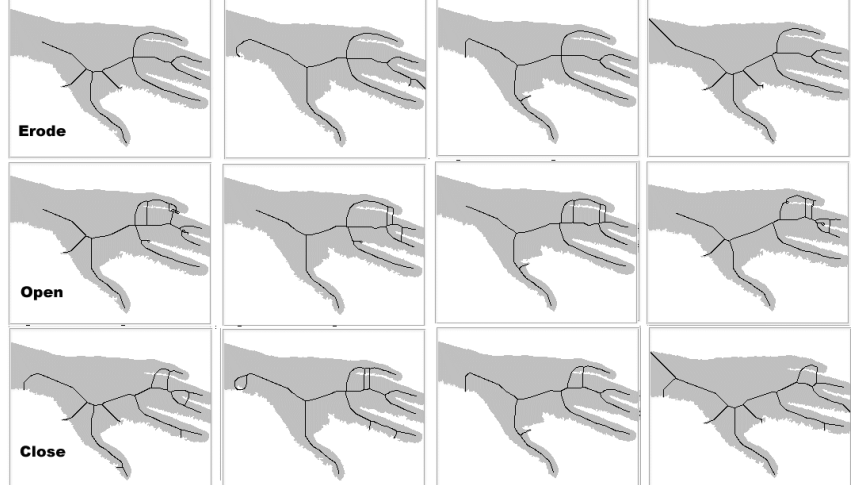

Figure 3 Morphological Preprocessing

Table 2 shows the stability of the skeletons when morphological preprocessing is applied. The erosion, opening and closing are denoted by "E-", "O-" and "C-" prefixes on the method labels in the left hand column.

Table 2 Morphological Preprocessing

\begin{tabular}{|l||c|c|c|c|c|c||c|}
\hline \multicolumn{1}{|c||}{} & \multicolumn{2}{c|}{ Rotation } & \multicolumn{2}{c|}{ Threshold } & \multicolumn{2}{c|}{ Noise } & Ave \\
\hline & $\mathrm{C}_{\mathrm{VB}}$ & $\mathrm{C}_{\mathrm{vJ}}$ & $\mathrm{C}_{\mathrm{vB}}$ & $\mathrm{C}_{\mathrm{vJ}}$ & $\mathrm{C}_{\mathrm{vB}}$ & $\mathrm{C}_{\mathrm{vB}}$ & $\mathrm{C}_{\mathrm{vJ}}$ \\
\hline E-EDF & 18.3 & 36.4 & 19.1 & 17.9 & 34.9 & 27.8 & 21.3 \\
\hline O-EDF & 17.4 & 49.0 & 22.3 & 20.6 & 40.5 & 25.5 & 27.9 \\
\hline C-EDF & 19.6 & 36.5 & 21.2 & 16.3 & 37.4 & 26.0 & 27.3 \\
\hline E-PAV & 21.8 & 21.7 & 29.0 & 27.4 & 60.8 & 59.7 & 29.7 \\
\hline O-PAV & 27.5 & 29.7 & 22.4 & 34.0 & 42.6 & 46.3 & 32.9 \\
\hline C-PAV & 20.3 & 22.1 & 23.9 & 23.6 & 72.6 & 47.5 & 31.0 \\
\hline E-ZHA & 18.6 & 20.2 & 24.0 & 27.6 & 64.6 & 58.5 & 31.8 \\
\hline O- ZHA & 25.2 & 26.7 & 18.4 & 33.7 & 49.5 & 38.1 & 32.0 \\
\hline C- ZHA & 18.4 & 24.5 & 16.4 & 19.9 & 58.1 & 51.7 & 31.2 \\
\hline
\end{tabular}

Comparing the results to Table 1 shows that erosion has little effect on the stability. Opening has the inverse effect of producing less stable skeletons. Closing produces similar instability to opening. While morphological preprocessing does not improve stability it does improve the visual quality of skeletons by reducing unwanted branches joins.

Table 3 Median Prefiltering (5x5 kernel)

\begin{tabular}{|c|c|c|c|c|c|c|c|}
\hline & \multicolumn{2}{|c|}{ Rotation } & \multicolumn{2}{|c|}{ Threshold } & \multicolumn{2}{|c|}{ Noise } & \multirow{2}{*}{$\frac{\text { Ave }}{c_{v J}}$} \\
\hline & $\mathrm{C}_{\mathrm{vB}}$ & $\mathrm{C}_{\mathrm{vJ}}$ & $\mathrm{C}_{\mathrm{vB}}$ & $\mathrm{C}_{\mathrm{vJ}}$ & $\mathrm{C}_{\mathrm{vB}}$ & $\mathrm{C}_{\mathrm{vB}}$ & \\
\hline EDF & 26.5 & 35.1 & 28.5 & 21.2 & 40.8 & 36.5 & 32.8 \\
\hline ROS & 17.8 & 29.9 & 20.7 & 28.8 & 47.2 & 42.8 & 32.4 \\
\hline PAV & 20.3 & 34.8 & 27.7 & 24.4 & 57.7 & 54.8 & 42.0 \\
\hline ZHA & 17.1 & 28.4 & 17.6 & 32.5 & 44.2 & 55.7 & 36.1 \\
\hline GUO & 18.8 & 24.6 & 13.5 & 23.2 & 60.5 & 36.5 & 27.3 \\
\hline FLUX & 33.3 & 30.8 & 19.7 & 27.5 & 29.8 & 24.8 & 28.2 \\
\hline FMM & 25.6 & 16.6 & 8.8 & 8.5 & 29.6 & 22.8 & 19.3 \\
\hline
\end{tabular}

Table 3 shows the stability of skeletons after preprocessing with a median filter. This also reduced the number of branches and joins but did not significantly change the stability. Figure 4 shows the skeletons produced for one of the sample images when using median pre-filtering for different MATs in each 
row. Each column shows the same test cases as in figure 3. As can be seen, the median prefiltering does not improve stability.

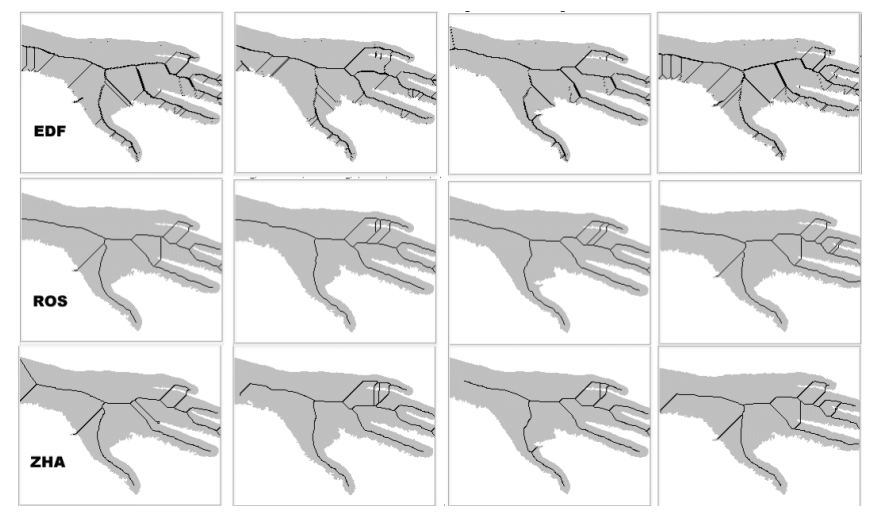

Figure 4 Median pre-filtering

Figure 5 shows the skeletons produced using shape peeling for the same test cases as in figure 2. While each MAT produces different skeletons, shape peeling is effective in suppressing spurious branches for all cases. The improvement in stability is noticeable for most methods but EDF. In this example the ROS and ZHA techniques are the most stable. While not as effective as the median filtering at suppressing spurs it came close on all but the EDF method.

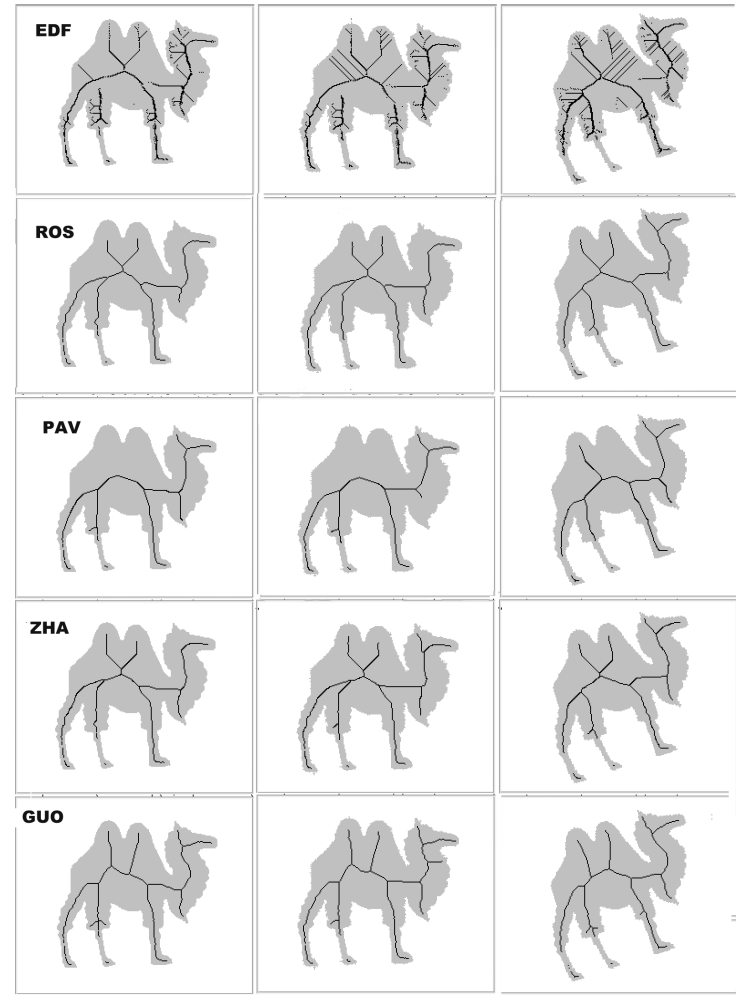

Figure 5 Shape Peeling Method

Table 4 presents the results for the skeleton stability using the shape peeling method. In this work the value of the peel thickness $\mathrm{T}_{\mathrm{d}}$ was set to 10 . More importantly it resulted in coefficients of variation in the range of $20 \%$ to $25 \%$ for all methods excluding the flux method. Comparing to table 1 the most significant improvement in stability was made to the thinning based methods with a negative improvement to the flux method. Shape peeling had little effect on the FMM based algorithm but significantly degraded the performance of the more computationally complex FLUX method.

Table 4 Shape Peeling

\begin{tabular}{|l||c|c|c|c|c|c||c|}
\hline \multicolumn{1}{|c||}{} & \multicolumn{2}{c|}{ Rotation } & \multicolumn{2}{c||}{ Threshold } & \multicolumn{2}{c|}{ Noise } & Ave \\
\hline & $\mathrm{C}_{\mathrm{vB}}$ & $\mathrm{C}_{\mathrm{vJ}}$ & $\mathrm{C}_{\mathrm{vB}}$ & $\mathrm{C}_{\mathrm{vJ}}$ & $\mathrm{C}_{\mathrm{vB}}$ & $\mathrm{C}_{\mathrm{vB}}$ & $\mathrm{C}_{\mathrm{vJ}}$ \\
\hline EDF & 23.4 & 25.4 & 12.3 & 14.7 & 23.6 & 20.9 & 20.1 \\
\hline ROS & 29.8 & 26.8 & 25.1 & 13.9 & 32.3 & 21.3 & 24.9 \\
\hline PAV & 31.8 & 19.7 & 28.3 & 14.9 & 31.6 & 16.6 & 23.8 \\
\hline ZHA & 30.6 & 22.9 & 24.5 & 16.7 & 31.5 & 17.4 & 23.9 \\
\hline GUO & 29.6 & 16.6 & 24.5 & 12.6 & 28.5 & 18.6 & 21.7 \\
\hline FLUX & 67.9 & 32.5 & - & - & 53.4 & 20.1 & 43.5 \\
\hline FMM & 27.7 & 16.0 & 26.1 & 15.2 & 25.7 & 15.1 & 21.0 \\
\hline
\end{tabular}

The computational efficiency of the shape peeling method compared to the base case and median filtering was evaluated as shown in Table 5. While typical preprocessing such as median filtering increases the computation time this is not the case with shape peeling. Rather, it increases the computational efficiency in most cases. This is partly due to the fact that peeling the shape boundary requires little computational overhead but reduces the amount of iterations required to deduce the skeleton. Hence the efficiency gain more than compensated for this overhead. The results presented in the table are in seconds and obtained on a MacBookPro running a 2.6GHz Intel i7 with $16 \mathrm{~GB}$ of $1600 \mathrm{MHz}$ DDR3 memory.

Table 5 Computational Efficiency (secs)

\begin{tabular}{|c||c|c|c|}
\hline & Base & Median (5x5) & Peeling \\
\hline EDF & 0.005 & 0.015 & 0.006 \\
\hline ROS & 0.031 & 0.046 & 0.031 \\
\hline PAV & 0.063 & 0.078 & 0.047 \\
\hline ZHA & 0.032 & 0.046 & 0.031 \\
\hline GUO & 0.078 & 0.094 & 0.078 \\
\hline FLUX & 10.656 & 9.109 & 8.018 \\
\hline FMM & 0.156 & 0.188 & 0.141 \\
\hline
\end{tabular}

\section{CONCLUSIONS}

Skeleton stability is an important requirement for skeleton based matching. This paper presented a study of the stability of medial axis transforms and various preprocessing techniques to improve it. The proposed shape peeling method was successfully able to significantly improve the stability of all but the best state of the art method. However, unlike that method it does not produce disjoint skeletons and typically requires around $1 / 3$ to $1 / 5$ the computational complexity to achieve the same level of stability when used in conjunction with an appropriate thinning algorithm. It is also at least 100 times faster and $50 \%$ more stable than the reportedly more robust, flux field based methods. 


\section{REFERENCES}

[1] H. Blum, A transformation for extracting new descriptors of shape, In Models for the Perception of Speech and Visual Form, W. Walthen-Dunn, editor, MIT Press, 1967.

[2] Per-Erik Danielsson, Euclidean distance mapping, Computer Graphics and Image Processing, Volume 14, Issue 3, November 1980, Pages 227-248

[3] A. Telea and J. J. van Wijk. 2002. "An augmented Fast Marching Method for computing skeletons and centerlines." In Proceedings of the symposium on Data Visualisation 2002 (VISSYM '02). Eurographics Association, Aire-la-Ville, Switzerland, 251-ff.

[4] L. Lam, S.W. Lee and C.Y. Suen, "Thinning Methodologies-AComprehensive Survey", IEEE Transactions on Pattern Analysis and Machine Intelligence, Vol. 14, No. 9, Sept. 1992, pp. 869-885

[5] Ogniewicz, R.; Ilg, M., "Voronoi skeletons: theory and applications,". Proceedings CVPR '92, 1992 IEEE Computer Society Conference on Computer Vision and Pattern Recognition, pp.63,69, 15-18 Jun 1992

[6] Dimitrov, P.; Phillips, C.; Siddiqi, K., "Robust and efficient skeletal graphs," Proceedings. IEEE Conference on, Computer Vision and Pattern Recognition, 2000. vol.1, no., pp.417,423 vol.1, 2000

[7] Cornea, N.D.; Silver, D.; Min, P., "Curve-Skeleton Properties, Applications, and Algorithms," Visualization and Computer Graphics, IEEE Transactions on, vol.13, no.3, pp.530,548, May-June 2007

[8] H. Chatbri, K. Kameyama "Towards making thinning algorithms robust against noise in sketch images" Pattern Recognition (ICPR), 2012 21st International Conference on , vol., no., pp.3030,3033, 11-15 Nov. 2012

[9] Kittler, J \& Illingworth, J (1986), "Minimum error thresholding", Pattern Recognition 19: 41-47

[10] Rosenfeld, A., "A characterization of parallel thinning algorithms," Information and Control, vol. 29, 1975, pp. 286-291

[11] T. Pavlidis. "A thinning algorithm for discrete binary images" Comput. Graphics Image Proc, 1980, 13:142157 ,

[12] Zhang \& Suen 1984 "A fast parallel algorithm for thinning digital patterns" Comms ACM. v.27, no.3, pp.236-239

[13 ] Z. Guo and R. Hall. "Parallel thinning with two subiteration algorithms" Commun. ACM 32, 3 (March 1989), 359-373.

[14] R. Zhou, H. C. Quek, and G. S. Ng. A novel singlepassthinning algorithm and an effective set of performance criteria. Pattern Recognition Letters, 16(12):1267-1275, 1995. 